Article

\title{
Acetic Acid Can Catalyze Succinimide Formation from Aspartic Acid Residues by a Concerted Bond Reorganization Mechanism: A Computational Study
}

\author{
Ohgi Takahashi *, Ryota Kirikoshi and Noriyoshi Manabe \\ Faculty of Pharmaceutical Sciences, Tohoku Pharmaceutical University, 4-4-1 Komatsushima, \\ Aoba-ku, Sendai 981-8558, Japan; E-Mails: kirikoshi@tohoku-pharm.ac.jp (R.K.); \\ manabe@tohoku-pharm.ac.jp (N.M.)
}

* Author to whom correspondence should be addressed; E-Mail: ohgi@tohoku-pharm.ac.jp; Tel.: +81-22-727-0208; Fax: +81-22-275-2013.

Academic Editor: Habil. Mihai V. Putz

Received: 13 December 2014 / Accepted: 4 January 2015 / Published: 12 January 2015

\begin{abstract}
Succinimide formation from aspartic acid (Asp) residues is a concern in the formulation of protein drugs. Based on density functional theory calculations using Ace-Asp-Nme $($ Ace $=$ acetyl, $\mathrm{Nme}=\mathrm{NHMe})$ as a model compound, we propose the possibility that acetic acid (AA), which is often used in protein drug formulation for mildly acidic buffer solutions, catalyzes the succinimide formation from Asp residues by acting as a proton-transfer mediator. The proposed mechanism comprises two steps: cyclization (intramolecular addition) to form a gem-diol tetrahedral intermediate and dehydration of the intermediate. Both steps are catalyzed by an AA molecule, and the first step was predicted to be rate-determining. The cyclization results from a bond formation between the amide nitrogen on the $C$-terminal side and the side-chain carboxyl carbon, which is part of an extensive bond reorganization (formation and breaking of single bonds and the interchange of single and double bonds) occurring concertedly in a cyclic structure formed by the amide $\mathrm{NH}$ bond, the AA molecule and the side-chain $\mathrm{C}=\mathrm{O}$ group and involving a double proton transfer. The second step also involves an AA-mediated bond reorganization. Carboxylic acids other than AA are also expected to catalyze the succinimide formation by a similar mechanism.
\end{abstract}


Keywords: aspartic acid residue; nonenzymatic reaction; succinimide; density functional theory; computational chemistry; acetic acid; buffer catalysis; double proton transfer; concerted bond reorganization; protein drugs

\section{Introduction}

Among nonenzymatic post-translational modifications of proteins, the alterations of normal L-aspartic acid (L-Asp) residues to L- $\beta$-Asp, D-Asp and D- $\beta$-Asp residues have recently attracted considerable attention because of relevance to aging and pathologies (especially those of age-related diseases, such as cataract and Alzheimer's disease) [1-12]. These altered Asp residues are formed through a five-membered cyclic succinimide intermediate having an aminosuccinyl (Asu) residue instead of Asp (Scheme 1) [13,14]. The L-Asu intermediate, having two carbonyl groups in the succinimide moiety, can be hydrolyzed either back to L-Asp or to L- $\beta$-Asp, the structural isomer of L-Asp. Moreover, because the succinimide is racemization-prone [15-17], D-Asp and D- $\beta$-Asp residues can also be formed via D-Asu.

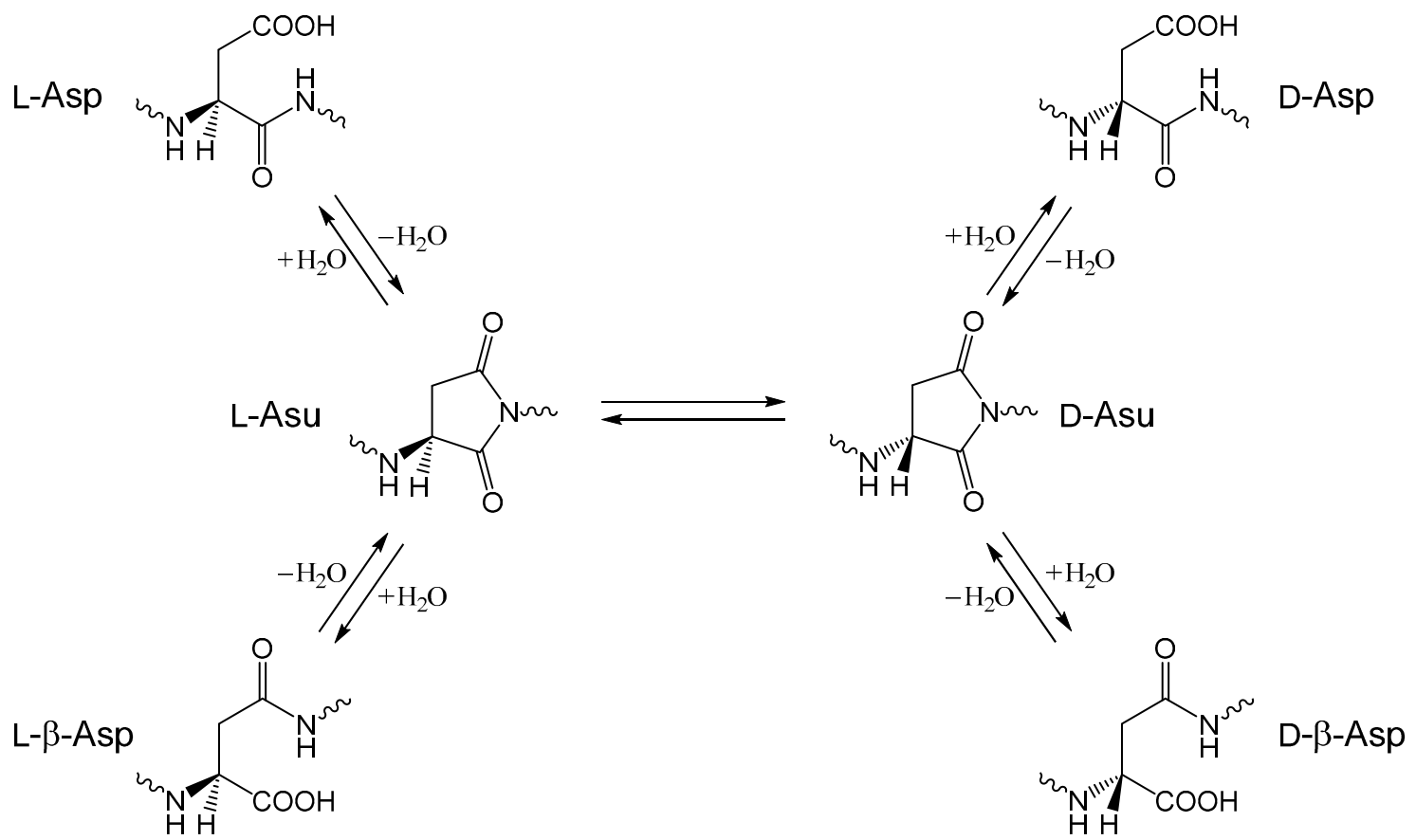

Scheme 1. Nonenzymatic reactions of aspartic acid (Asp) residues via the succinimide intermediate (aminosuccinyl (Asu) residue).

Succinimide formation has been regarded as a nucleophilic substitution at the carbonyl carbon comprised of two steps (an addition-elimination or a cyclization-dehydration mechanism) (Scheme 2) [18-20]. The first step is an intramolecular addition (cyclization) in which the amide nitrogen of the $C$-terminal peptide bond nucleophilically attacks the carboxyl carbon of the Asp side chain. This gives a tetrahedral intermediate, which is probably a gem-diol at neutral to acidic $\mathrm{pH}[20,21]$. In the second step, a water molecule is eliminated from the gem-diol group. Both steps are thought to require a catalyst, because density-functional quantum-chemical calculations show that the energy barriers are too high without 
a catalyst [20,21]. Water is a good candidate as a catalyst of the succinimide-forming reactions, as we have recently shown computationally [22-24].

Buffers may also catalyze the succinimide-forming reactions. When hen egg-white lysozyme was incubated at $\mathrm{pH} 4.0$ (acetate buffer) and at $40{ }^{\circ} \mathrm{C}$, conversion of the Asp101 residue to the Asu form was observed [25]. The formation of the Asu residue increased and saturated as the concentration of acetate buffer was increased. This may indicate that acetic acid (AA) or acetate ion acts as a catalyst in succinimide formation. It should be noted that Asp101 is located in a structurally flexible region on the surface of the lysozyme molecule; in particular, its side chain is conformationally disordered and also the carboxyl group is highly solvent-accessible [26]. It is also notable that the Asu-containing lysozyme can be isolated as crystals [27,28].

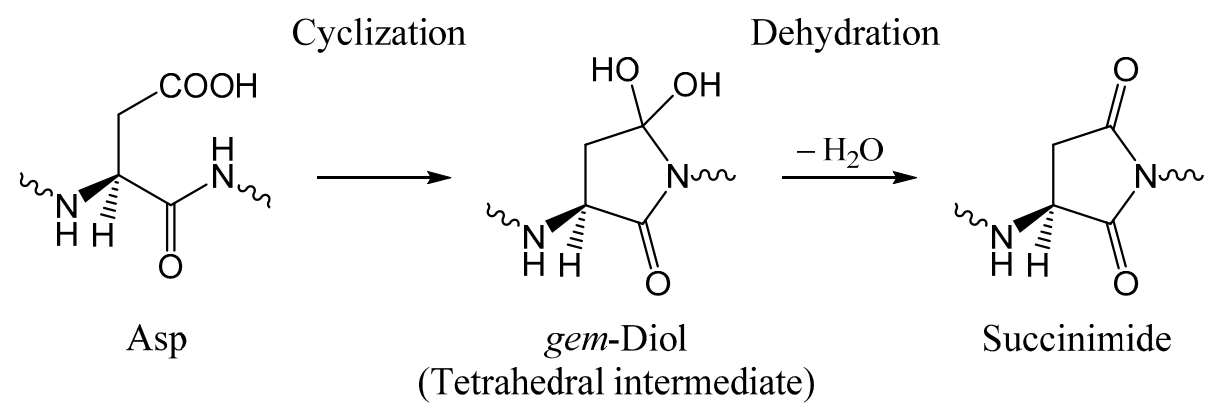

Scheme 2. Two-step mechanism for succinimide formation from an Asp residue.

From a mechanistic point of view, there is another concern. Amide nitrogens are thought to be poor nucleophiles, because of the amide resonance (conjugation with the electron-withdrawing carbonyl group) [29]. Indeed, nucleophilic attacks by amide nitrogens are not very common in synthetic organic chemistry [30-34]. Can the nucleophilic attacks by amide nitrogens really occur under mild conditions? Recently, we have computationally shown the possibility that cyclization to give a gem-diol tetrahedral intermediate occurs after the amide group is converted to the tautomeric iminol form [22-24]. The $\pi$ electron density of the nitrogen atom is thought to be increased by iminolization, enhancing its nucleophilicity.

The alterations of L-Asp residues also occur in protein drugs, such as monoclonal antibodies [35-49], which may affect their stability, potency and/or safety, presenting challenges to the pharmaceutical industry during the process of protein purification, formulation, storage and delivery. Mildly acidic buffers have been widely used for the formulation of proteins, because many physical and chemical changes tend to be minimized at $\mathrm{pH} 4-5$ [50,51]. However, it has been noticed that the succinimide intermediate is more stable (or its formation is faster) at mildly acidic $\mathrm{pH}$ than at higher $\mathrm{pH}[18,41,42,45,47,52-54]$. Moreover, since AA is commonly used for mildly acidic buffers, the above-mentioned possibility of AA-catalyzed succinimide formation from Asp residues could be a pharmaceutical concern. On the other hand, $\alpha$-aminosuccinimides themselves have received considerable attention in drug design [55].

In this paper, we computationally show that an AA molecule $\left(\mathrm{CH}_{3} \mathrm{COOH}\right.$, not $\left.\mathrm{CH}_{3} \mathrm{COO}^{-}\right)$can catalyze the two-step formation of succinimide from L-Asp residues (Scheme 2). As in our previous studies [22-24], Ace-Asp-Nme (Ace = acetyl, Nme = NHMe) (Figure 1) was employed as a model compound. Note that the Asp side chain is in the protonated form $(-\mathrm{COOH})$, because only this form is 
thought to undergo nucleophilic attack by the backbone nitrogen atom to form the five-membered ring $[14,18,47,49]$. Although Asp residues exist essentially in the deprotonated form $\left(-\mathrm{COO}^{-}\right)$at neutral or physiological $\mathrm{pH}$, the amount of the protonated form increases as $\mathrm{pH}$ decreases.

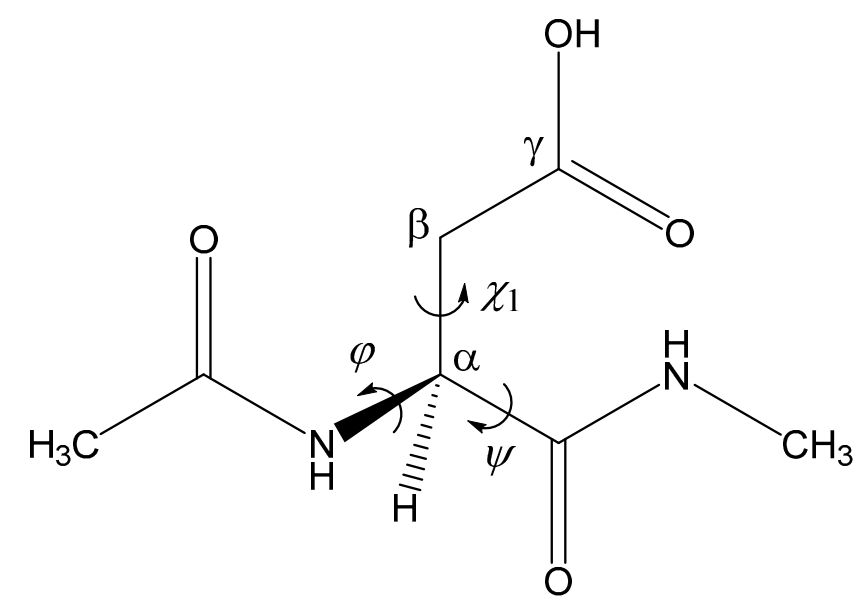

Figure 1. The model compound used in the present study (Ace-Asp-Nme). The $\varphi$ $\left(\mathrm{C}-\mathrm{N}-\mathrm{C}_{\alpha}-\mathrm{C}\right)$ and $\psi\left(\mathrm{N}-\mathrm{C}_{\alpha}-\mathrm{C}-\mathrm{N}\right)$ dihedral angles, which characterize the main-chain conformation, and the $\chi_{1}$ dihedral angle $\left(\mathrm{N}-\mathrm{C}_{\alpha}-\mathrm{C}_{\beta}-\mathrm{C}_{\gamma}\right)$, which characterizes the side-chain conformation, are indicated.

\section{Results and Discussion}

Figure 2 shows the energy diagram for the two-step succinimide formation catalyzed by an AA molecule, and Figures 3-9 show optimized geometries. The values of dihedral angles $\varphi, \psi$ and $\chi_{1}$ (Figure 1) are shown in the captions to Figures 3-9. R, I and P stand for the reactant (model compound), the gem-diol tetrahedral intermediate and the succinimide product, respectively. AA and $\mathrm{W}$ are acetic acid and water molecules, respectively. TS- 1 and TS-2 are the transition states of the first and second steps (cyclization and dehydration), respectively. While geometry optimizations and zero-point energy (ZPE) calculations were performed in a vacuum, hydration free energies estimated by the SM8 (solvation model 8) continuum model [56,57] were taken into account in relative energy calculations. As may be seen from Figure 2, the effects of hydration on relative energies are small, except for complexation energies. The relative energies cited in the following are those in water, unless otherwise noted.

The reactant molecule $\mathrm{R}$ shown in Figure $3 \mathrm{a}$ is in an extended conformation with the backbone dihedral angles $\varphi$ and $\psi$ (Figure 1) being $-162^{\circ}$ and $162^{\circ}$, respectively. Note that this is not the most stable conformer, but the "reactive" conformer, in that it can form the reactant complex R•AA (a 1:1 complex between R and AA) (Figure 4), from which cyclization to the five-membered ring occurs. In R•AA, the AA molecule forms two hydrogen bonds to R, bridging the $\mathrm{NH}$ of the $C$-terminal peptide bond and the $\mathrm{C}=\mathrm{O}$ of the side-chain carboxyl group. As a result, the distance between the $\mathrm{NH}$ nitrogen and the carboxyl carbon is $3.367 \AA$. Upon complexation between $\mathrm{R}$ and AA, the dihedral angle $\psi$ changes by $20^{\circ}$, while changes in $\varphi$ and $\chi_{1}$ are much smaller. The complexation energies calculated in a vacuum and in water are 11.9 and $4.0 \mathrm{kcal} \cdot \mathrm{mol}^{-1}$, respectively. 


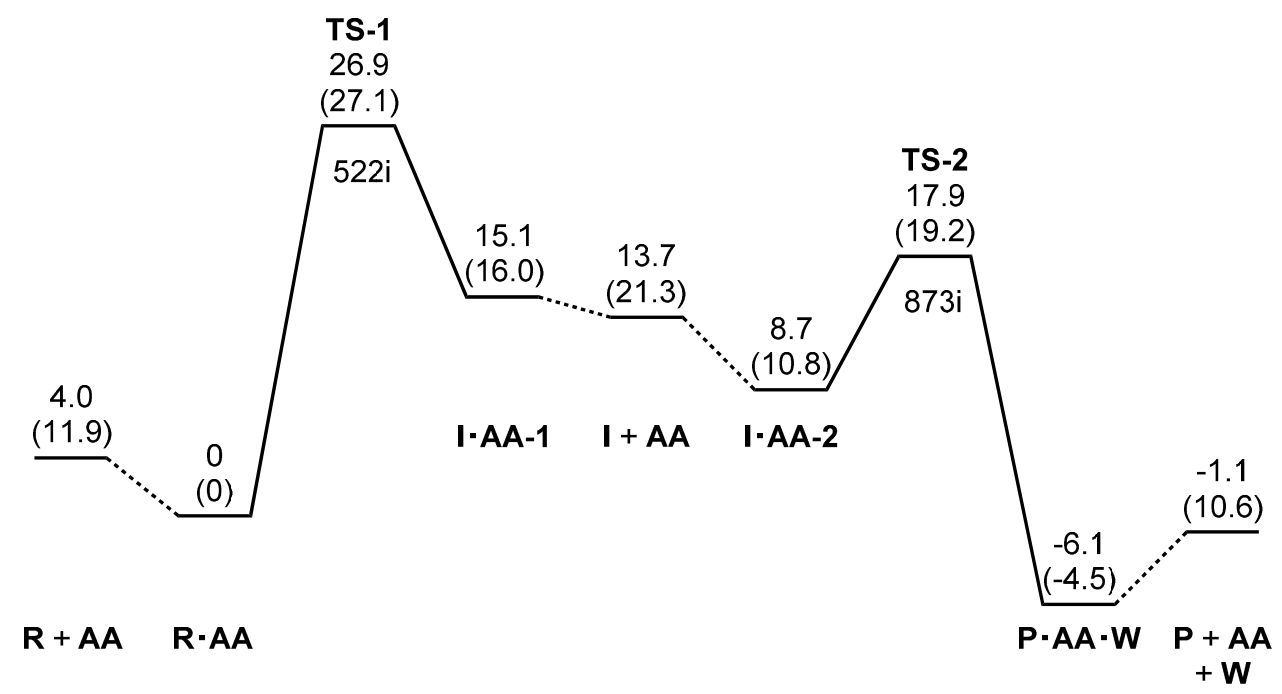

Figure 2. Energy diagram $\left(\mathrm{kcal} \cdot \mathrm{mol}^{-1}\right)$, where relative energies corrected for the zero-point energy (ZPE) and the SM8 (solvation model 8) hydration free energy are shown with respected to the reactant complex, $\mathrm{R} \cdot \mathrm{AA}$ (R, reactant molecule; AA, acetic acid). The ZPE-corrected relative energies in a vacuum are shown in parentheses for comparison. The single imaginary frequency $\left(\mathrm{cm}^{-1}\right)$ is also shown for TS-1 and TS-2 (TS, transition state). I, intermediate; $\mathrm{P}$, product molecule; $\mathrm{W}$, water.
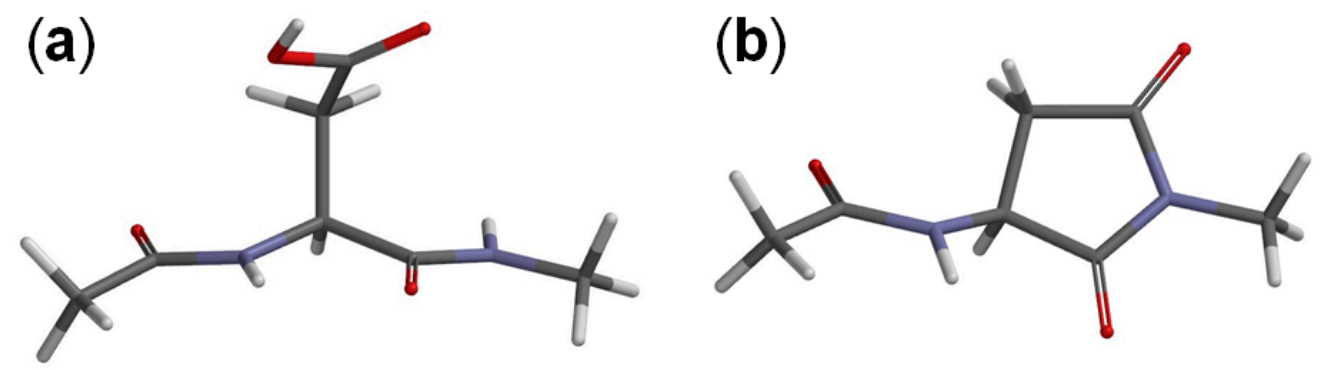

Figure 3. The geometries of (a) the reactant $R$ (model compound, Figure 1) $\left(\varphi=-162^{\circ}\right.$, $\left.\psi=162^{\circ}, \chi_{1}=72^{\circ}\right)$ and $(\mathbf{b})$ the succinimide product $\mathrm{P}\left(\varphi=-171^{\circ}, \psi=-141^{\circ}, \chi_{1}=136^{\circ}\right)$.

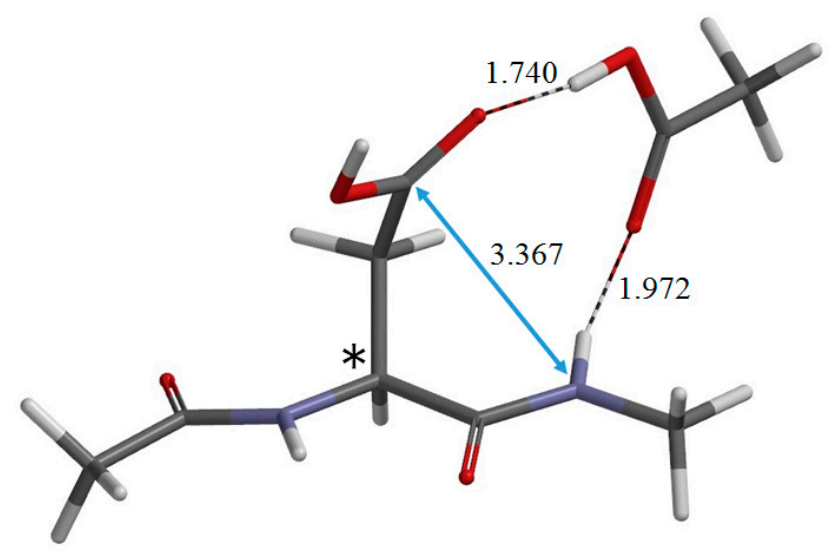

Figure 4. The geometry of the reactant complex R・AA $\left(\varphi=-164^{\circ}, \psi=-178^{\circ}, \chi_{1}=76^{\circ}\right)$. The $\alpha$ carbon atom is indicated by an asterisk $(*)$. Selected interatomic distances are shown in $\AA$. The gas-phase total energy of this geometry is $-913.600369 E_{\mathrm{h}}$. 
TS-1 (Figure 5) is the transition state for cyclization from the reactant complex $\mathrm{R} \cdot \mathrm{AA}$, and I•AA-1 (Figure 6) is the intermediate complex directly connected to TS-1. The distance of the forming $\mathrm{C}-\mathrm{N}$ bond in TS-1 is $1.755 \AA$, and that of the newly formed $\mathrm{C}-\mathrm{N}$ bond in I•AA-1 is $1.495 \AA$. Because of the intramolecular cyclization, changes in $\psi$ and $\chi_{1}$ by about $40^{\circ}$ occur continuously from $\cdot \mathrm{AA}^{\mathrm{A}}$ to I•AA-1 through TS-1.

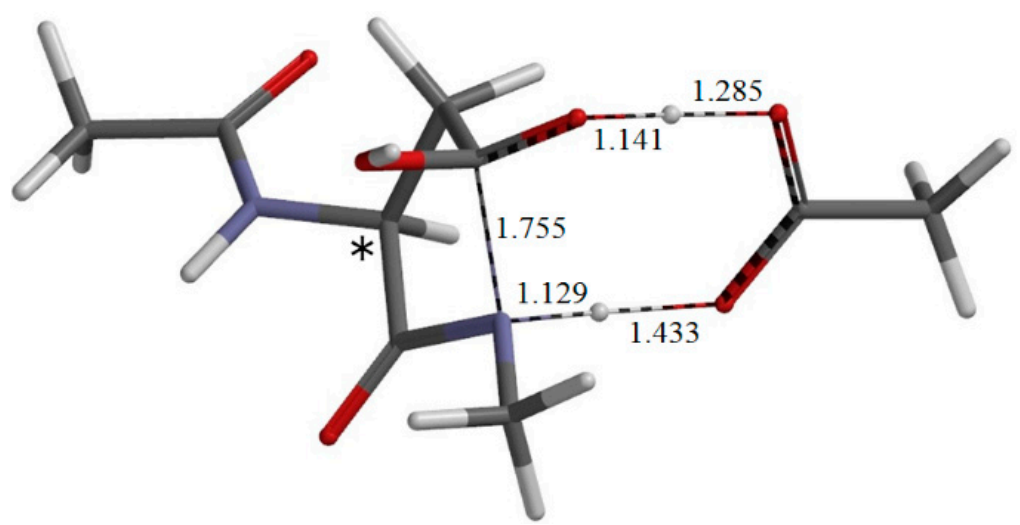

Figure 5. The geometry of the transition state TS-1 of the first step (cyclization) $\left(\varphi=-162^{\circ}\right.$, $\left.\psi=-150^{\circ}, \chi_{1}=115^{\circ}\right)$. The distances of forming and breaking bonds are shown in $\AA$. The asterisk $(*)$ indicates the $\alpha$ carbon.

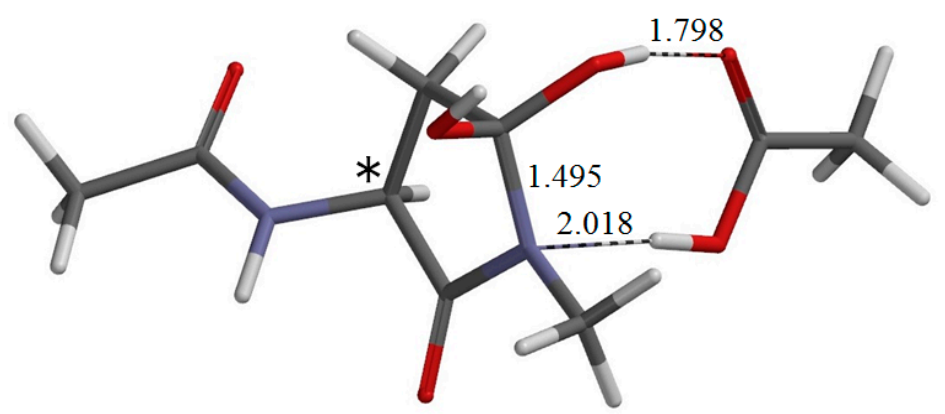

Figure 6. The geometry of I•AA-1 $\left(\varphi=-167^{\circ}, \psi=-138^{\circ}, \chi_{1}=117^{\circ}\right)$, which is the intermediate complex directly connected to TS-1. Selected interatomic distances are shown in $\AA$. The asterisk (*) indicates the $\alpha$ carbon.

Concomitantly with the $\mathrm{C}-\mathrm{N}$ bond formation, a double proton transfer mediated by the AA molecule occurs, so that the resultant intermediate species is a gem-diol having two $\mathrm{OH}$ groups on the $\mathrm{C}_{\gamma}$ atom. More specifically, the $\mathrm{NH}$ hydrogen moves toward the $\mathrm{C}=\mathrm{O}$ oxygen of $\mathrm{AA}$, the $\mathrm{OH}$ hydrogen of $\mathrm{AA}$ moves toward the $\mathrm{C}=\mathrm{O}$ oxygen of the side chain and the single and double bonds are interchanged in the $\mathrm{COO}$ moiety of AA. The AA molecule thus acts as both proton donor and acceptor in the double proton transfer. Moreover, this double proton transfer is somewhat asynchronous; namely, the proton transfer from the AA molecule precedes that from the NH group. In the resultant complex, I•AA-1, there are two hydrogen bonds between I (the intermediate in its isolated state; geometry not shown) and AA. One is between the amide nitrogen in the five-membered ring and the $\mathrm{OH}$ of the newly-formed $\mathrm{AA}$. The other is between the $\mathrm{C}=\mathrm{O}$ of $\mathrm{AA}$ and the newly-formed $\mathrm{OH}$ in the gem-diol group. 
The energy of TS-1 relative to the reactant complex R•AA was calculated to be $26.9 \mathrm{kcal} \cdot \mathrm{mol}^{-1}$ in water. This value is higher than that of TS-2 (see below) by $9 \mathrm{kcal} \cdot \mathrm{mol}^{-1}$. Therefore, the first step is predicted to be the rate-determining step. Moreover, the value of $26.9 \mathrm{kcal} \cdot \mathrm{mol}^{-1}$ is very close to the activation barrier recently calculated for a three-water-catalyzed succinimide formation from Asp $\left(26.7 \mathrm{kcal} \cdot \mathrm{mol}^{-1}\right)$ [24] and is plausible for a nonenzymatic reaction, which occurs slowly at room temperature or physiological temperature.

In a vacuum, the complex I•AA-1 is more stable than the separated state $(\mathrm{I}+\mathrm{AA})$ by about $5 \mathrm{kcal} \cdot \mathrm{mol}^{-1}$. However, it becomes less stable than the separated state by $1.4 \mathrm{kcal} \cdot \mathrm{mol}^{-1}$ when the hydration effect is taken into account. Moreover, there exists another intermediate complex (I•AA-2, Figure 7), which is much more stable than I•AA-1, both in a vacuum and in water. In water, I•AA-2 is more stable than $\mathrm{I} \bullet \mathrm{AA}-1$ by $6.4 \mathrm{kcal} \cdot \mathrm{mol}^{-1}$ and higher in energy than $\mathrm{R} \cdot \mathrm{AA}$ by $8.7 \mathrm{kcal} \cdot \mathrm{mol}^{-1}$. In $\mathrm{I} \cdot \mathrm{AA}-2$, the AA molecule forms two hydrogen bonds with the gem-diol group of I (Figure 7). The $\mathrm{C}=\mathrm{O}$ oxygen of AA forms a hydrogen bond to one of the $\mathrm{OH}$ groups in the gem-diol moiety, and the $\mathrm{OH}$ hydrogen of AA forms a hydrogen bond to the oxygen of the other $\mathrm{OH}$ group in the gem-diol moiety. I•AA-1 and $\mathrm{I} \bullet \mathrm{AA}-2$ have similar main-chain conformations, but $\chi_{1}$ is larger in I•AA-2 by $29^{\circ}$ than in I•AA-1.

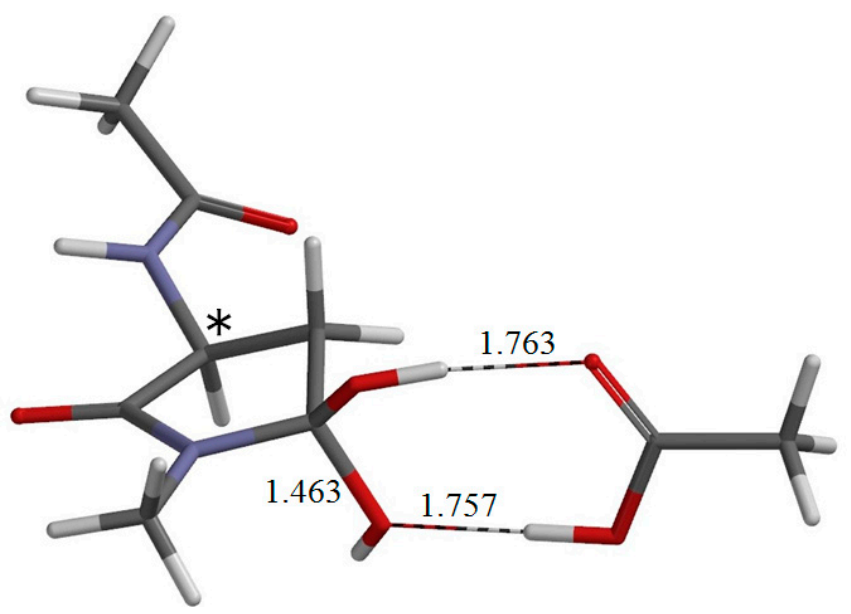

Figure 7. The geometry of $\mathrm{I} \bullet \mathrm{AA}-2\left(\varphi=-170^{\circ}, \psi=-145^{\circ}, \chi_{1}=146^{\circ}\right)$, which is the intermediate complex directly connected to TS-2. Selected interatomic distances are shown in $\AA$. The asterisk (*) indicates the $\alpha$ carbon.

From I•AA-2, dehydration occurs via the transition state TS-2 (Figure 8) to give the product complex $\mathrm{P} \bullet \mathrm{AA} \bullet \mathrm{W}$ (Figure 9). In this step, one of the $\mathrm{C}-\mathrm{O}$ bonds in the gem-diol moiety is cleaved (see Figure 8). In TS-2, the breaking $\mathrm{C}-\mathrm{O}$ bond is elongated to $1.837 \AA$ from $1.463 \AA$ in I•AA-2. Concomitantly with this bond cleavage, a double proton transfer occurs mediated by the AA molecule. The $\mathrm{OH}$ hydrogen of AA moves toward the departing oxygen, leading to the formation of a water molecule. On the other hand, the hydrogen attached to the other oxygen of the gem-diol moiety moves toward the $\mathrm{C}=\mathrm{O}$ oxygen of AA. In this process, the AA molecule again acts as both proton donor and acceptor. It should be noted that the local activation barrier of the second step is as low as $9.2 \mathrm{kcal} \cdot \mathrm{mol}^{-1}$. The relative energy of TS-2 with respect to $\mathrm{R} \cdot \mathrm{AA}$ is $17.9 \mathrm{kcal} \cdot \mathrm{mol}^{-1}$, which is lower than that of TS- 1 by $9 \mathrm{kcal} \cdot \mathrm{mol}^{-1}$. The resultant $\mathrm{P} \bullet \mathrm{AA} \bullet \mathrm{W}$ is a complex formed by $\mathrm{P}$ (Figure $3 \mathrm{~b}$ ), $\mathrm{AA}$ and $\mathrm{W}$ and is more stable than the separated state $(\mathrm{P}+\mathrm{AA}+\mathrm{W})$ by 15.1 and $5.0 \mathrm{kcal} \cdot \mathrm{mol}^{-1}$ in a vacuum and in water, respectively. The changes in $\varphi$, 
$\psi$ and $\chi_{1}$ continuously from $\mathrm{I} \bullet \mathrm{AA}-2$ to $\mathrm{P} \bullet \mathrm{AA} \bullet \mathrm{W}$ are very small. The energy of $\mathrm{P} \bullet \mathrm{AA} \bullet \mathrm{W}$ is lower than that of $\mathrm{I} \cdot \mathrm{AA}-2$ by $14.8 \mathrm{kcal} \cdot \mathrm{mol}^{-1}$.

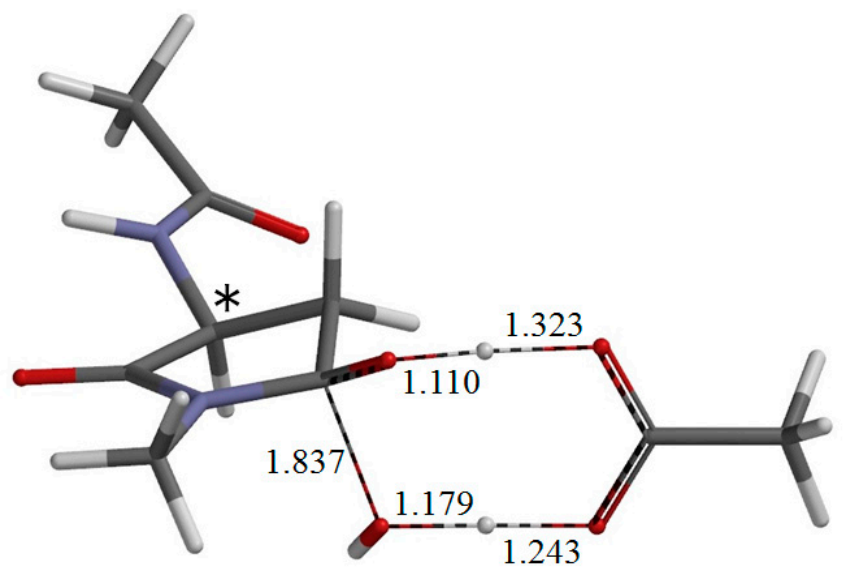

Figure 8. The geometry of the transition state TS-2 of the second step (dehydration) $\left(\varphi=-169^{\circ}, \psi=-142^{\circ}, \chi_{1}=144^{\circ}\right)$. The distances of forming and breaking bonds are shown in $\AA$. The asterisk (*) indicates the $\alpha$ carbon.

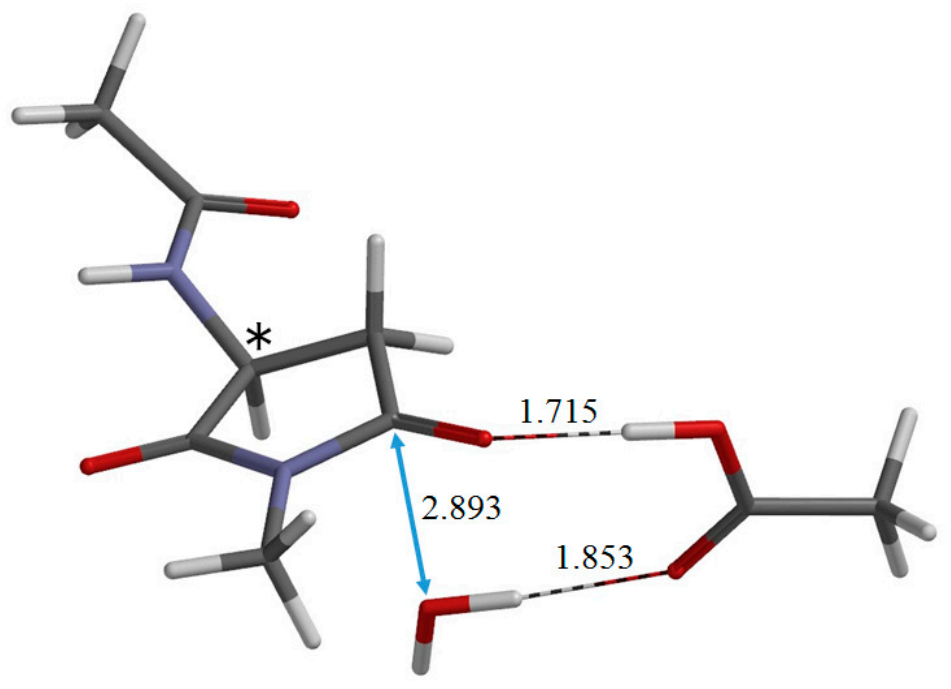

Figure 9. The geometry of the product complex $\mathrm{P} \cdot \mathrm{AA} \bullet \mathrm{W}\left(\varphi=-173^{\circ}, \psi=-140^{\circ}, \chi_{1}=137^{\circ}\right)$ formed by the succinimide product $\mathrm{P}$, the regenerated acetic acid molecule AA and the released water molecule W. Selected interatomic distances are shown in $\AA$. The asterisk (*) indicates the $\alpha$ carbon.

When the energies of the initial separated state $(\mathrm{R}+\mathrm{AA})$ and the final separated state $(\mathrm{P}+\mathrm{AA}+\mathrm{W})$ are compared, the latter was calculated to be more stable by $5.1 \mathrm{kcal} \cdot \mathrm{mol}^{-1}$. Considering that the experimental hydration free energy of water is $-6.3 \mathrm{kcal} \cdot \mathrm{mol}^{-1}$ [58], while it is calculated to be $-9.1 \mathrm{kcal} \cdot \mathrm{mol}^{-1}$ at the present level of calculation, it may be said that the reactant and product states are very close to each other in energy. This is consistent with the fact that the Asp and Asu forms coexist in aqueous solution depending on the conditions [18,25,38,54].

The double proton transfers in the first and second steps, especially the latter, may remind one of the degenerate, synchronous double proton transfers occurring in the cyclic dimers of carboxylic acids, 
including the AA dimer [59-61]. The best theoretical estimate of the barrier height for the double proton transfer in the formic acid dimer is $8.94 \mathrm{kcal} \cdot \mathrm{mol}^{-1}$ [59]. This value can be compared with the local activation barrier for the second step of the present reaction $\left(8.4\right.$ and $9.2 \mathrm{kcal} \cdot \mathrm{mol}^{-1}$ in a vacuum and in water, respectively). It should also be noted that carboxylic acids participate in many excited-state double-proton transfer (ESDPT) processes [62-65].

From the viewpoint of mechanistic organic chemistry, both the first and second steps can be viewed as a bond reorganization process occurring in a cyclic hydrogen-bonded complex. The first step (cyclization), in particular, has been regarded as a nucleophilic attack by the amide nitrogen. Although this is more or less correct, it may be better viewed as a concerted bond reorganization process, which bears some resemblance to pericyclic reactions, considering that amide nitrogens are generally recognized as poor nucleophiles.

\section{Computational Details}

Figure 1 shows the model compound used in the present study, in which an Asp residue is capped with Ace and Nme groups on the $N$ - and $C$-termini, respectively. This compound has previously been used in related computational studies by us [22-24] and Catak et al. [21,66]. The two-step reaction pathway was explored for a reactant complex formed between the model compound and a catalytic AA molecule.

All calculations were performed by using Spartan'14 [67]. As in our previous studies [22-24], energy-minimum and transition state geometries were located in a vacuum without any constraints by the density functional theory (DFT) with the B3LYP functional and the 6-31+G(d,p) basis set. Vibrational frequency calculations were performed for all of the optimized geometries to confirm them as energy minima (with no imaginary frequency) or transition states (with a single imaginary frequency) and to correct the relative energies for ZPE. Intrinsic reaction coordinate (IRC) calculations were performed from the transition states followed by full geometry optimizations to confirm that each transition state connects two energy minima, as shown in Figure 2. Furthermore, hydration effects have been included by single-point calculations at the same level of theory employing the SM8 continuum model [56,57].

\section{Conclusions}

We have computationally shown that acetic acid (AA) $\left(\mathrm{CH}_{3} \mathrm{COOH}\right.$, not $\left.\mathrm{CH}_{3} \mathrm{COO}^{-}\right)$can catalyze succinimide formation from Asp residues. The reaction proceeds by two steps, cyclization and dehydration, and an AA molecule catalyzes both steps by acting as both proton donor and acceptor in double-proton transfers. The size and shape of the carboxyl group $(-\mathrm{COOH})$ seem to be almost perfect to enable the double-proton transfer, both in the cyclization and dehydration steps. The rate-determining step was predicted to be the cyclization step (first step). Since protein drugs, especially monoclonal antibodies, are often formulated in acetate buffer, the AA-catalyzed reaction as revealed here can be a pharmaceutical concern. Carboxylic acids other than AA are also expected to catalyze succinimide formation from Asp residues. Very recently, deamidation of asparagine (Asn) residues (which also proceeds via the succinimide intermediate) has been shown to be catalyzed by a variety of carboxylic acids [68]. A similar mechanism to the presently proposed one may also operate in the Asn deamidation 
reactions. From the viewpoint of mechanistic organic chemistry, the "nucleophilic" attack by the amide nitrogen of the peptide backbone may be viewed as nominal. The cyclization step may better be viewed as a concerted bond reorganization process.

\section{Author Contributions}

Ohgi Takahashi performed the DFT calculations and wrote the manuscript. All of the authors examined and discussed the results and approved the final version.

\section{Conflicts of Interest}

The authors declare no conflict of interest.

\section{References}

1. Ritz-Timme, S.; Collins, M.J. Racemization of aspartic acid in human proteins. Aging Res. Rev. 2002, 1, 43-59.

2. Clarke, S. Aging as war between chemical and biochemical processes: Protein methylation and the recognition of age-damaged proteins for repair. Aging Res. Rev. 2003, 2, 263-285.

3. Fujii, N. D-Amino acid in elderly tissues. Biol. Pharm. Bull. 2005, 28, 1585-1589.

4. Truscott, R.J.W. Are ancient proteins responsible for the age-related decline in health and fitness? Rejuvenation Res. 2010, 13, 83-89.

5. Fujii, N.; Takemoto, L.J.; Momose, Y.; Matsumoto, S.; Hiroki, K.; Akaboshi, M. Formation of four isomers at the Asp-151 residue of aged human $\alpha \mathrm{A}$-crystallin by natural aging. Biochem. Biophys. Res. Commun. 1999, 265, 746-751.

6. Fujii, N.; Matsumoto, S.; Hiroki, K.; Takemoto, L. Inversion and isomerization of Asp-58 residue in human $\alpha \mathrm{A}$-crystallin from normal aged lenses and cataractous lenses. Biochim. Biophys. Acta 2001, 1549, 179-187.

7. Fujii, N.; Kawaguchi, T.; Sasaki, H.; Fujii, N. Simultaneous stereoinversion and isomerization at the Asp-4 residue in $\beta \mathrm{B} 2$-crystallin from the aged human eye lenses. Biochemistry 2011, 50, 8628-8635.

8. Hooi, M.Y.S.; Truscott, R.J.W. Racemisation and human cataract: D-Ser, D-Asp/Asn and D-Thr are higher in the lifelong proteins of cataract lenses than in age-matched normal lenses. Age 2011, 33, 131-141.

9. Roher, A.E.; Lowenson, J.D.; Clarke, S.; Wolkow, C.; Wang, R.; Cotter, R.J.; Reardon, I.M.; Zürcher-Neely, H.A.; Heinrikson, R.L.; Ball, M.J.; et al. Structural alterations in the peptide backbone of $\beta$-amyloid core protein may account for its deposition and stability in Alzheimer's disease. J. Biol. Chem. 1993, 268, 3072-3083.

10. Tomiyama, T.; Asano, S.; Furiya, Y.; Shirasawa, T.; Endo, N.; Mori, H. Racemization of Asp ${ }^{23}$ residue affects the aggregation properties of Alzheimer amyloid $\beta$ protein analogues. J. Biol. Chem. 1994, 269, 10205-10208. 
11. Sadakane, Y.; Konoha, K.; Kawahara, M.; Nakagomi, K. Quantification of structural alterations of L-Asp and L-Asn residues in peptides related to neuronal diseases by reversed-phase high-performance liquid chromatography. Chem. Biodivers. 2010, 7, 1371-1379.

12. Galletti, P.; de Bonis, M.L.; Sorrentino, A.; Raimo, M.; D’Angelo, S.; Scala, I.; Andria, G.; D’Aniello, A.; Ingrosso, D.; Zappia, V. Accumulation of altered aspartyl residues in erythrocyte proteins from patients with Down's syndrome. FEBS J. 2007, 274, 5263-5277.

13. Geiger, T.; Clarke, S. Deamidation, isomerization, and racemization at asparaginyl and aspartyl residues in peptides: Succinimide-linked reactions that contribute to protein degradation. J. Biol. Chem. 1987, 262, 785-794.

14. Stephenson, R.C.; Clarke, S. Succinimide formation from aspartyl and asparaginyl peptides as a model for the spontaneous degradation of proteins. J. Biol. Chem. 1989, 264, 6164-6170.

15. Radkiewicz, J.L.; Zipse, H.; Clarke, S.; Houk, K.N. Accelerated racemization of aspartic acid and asparagine residues via succinimide intermediates: An ab initio theoretical exploration of mechanism. J. Am. Chem. Soc. 1996, 118, 9148-9155.

16. Takahashi, O.; Kobayashi, K.; Oda, A. Modeling the enolization of succinimide derivatives, a key step of racemization of aspartic acid residues: Importance of a two- $\mathrm{H}_{2} \mathrm{O}$ mechanism. Chem. Biodivers. 2010, 7, 1349-1356.

17. Takahashi, O. Two-water-assisted racemization of the succinimide intermediate formed in proteins: A computational model study. Health 2013, 5, 2018-2021.

18. Capasso, S. Thermodynamic parameters of the reversible isomerization of aspartic residues via a succinimide derivative. Thermochim. Acta 1996, 286, 41-50.

19. Reissner, K.J.; Aswad, D.W. Deamidation and isoaspartate formation in proteins: Unwanted alterations or surreptitious signals? Cell. Mol. Life Sci. 2003, 60, 1281-1295.

20. Aylin, F.; Konuklar, S.; Aviyente, V. Modelling the hydrolysis of succinimide: Formation of aspartate and reversible isomerization of aspartic acid via succinimide. Org. Biomol. Chem. 2003, 1, 2290-2297.

21. Catak, S.; Monard, G.; Aviyente, V.; Ruiz-López, M.F. Deamidation of asparagine residues: Direct hydrolysis versus succinimide-mediated deamidation mechanisms. J. Phys. Chem. A 2009, 113, 1111-1120.

22. Takahashi, O.; Oda, A. Amide-iminol tautomerization of the $C$-terminal peptide groups of aspartic acid residues: Two-water-assisted mechanism, cyclization from the iminol tautomer leading to the tetrahedral intermediate of succinimide formation, and implication to peptide group hydrogen exchange. In Tyrosine and Aspartic Acid: Properties, Sources and Health Benefits; Jones, J.E., Morano, D.M., Eds.; Nova Science Publishers: New York, NY, USA, 2012; pp. 131-147.

23. Takahashi, O.; Kirikoshi, R. Intramolecular cyclization of aspartic acid residues assisted by three water molecules: A density functional theory study. Comput. Sci. Discov. 2014, 7, 015005.

24. Takahashi, O.; Kirikoshi, R.; Manabe, N. Roles of intramolecular and intermolecular hydrogen bonding in a three-water-assisted mechanism of succinimide formation from aspartic acid residues. Molecules 2014, 19, 11440-11452.

25. Tomizawa, H.; Yamada, H.; Ueda, T.; Imoto, T. Isolation and characterization of 101-succinimide lysozyme that possesses the cyclic imide at Asp101-Gly102. Biochemistry 1994, 33, 8770-8774. 
26. Vaney, M.C.; Maignan, S.; Riès-Kautt, M.; Ducruix, A. High-resolution structure (1.33 Å) of a HEW lysozyme tetragonal crystal grown in the APCF apparatus: Data and structural comparison with a crystal grown under microgravity from SpaceHab-01 mission. Acta Crystallogr. D Biol. Crystallogr. 1996, 52, 505-517.

27. Miyawaki, K.; Noguchi, S.; Harada, S.; Satow, Y. Crystallography of succinimide hen egg-white lysozyme at low temperatures. J. Cryst. Growth 1996, 168, 292-296.

28. Noguchi, S.; Miyawaki, K.; Satow, Y. Succinimide and isoaspartate residues in the crystal structures of hen egg-white lysozyme complexed with tri- $N$-acetylchitotriose. J. Mol. Biol. 1998, 278, 231-238.

29. Jacobs, A. Understanding Organic Reaction Mechanisms; Cambridge University Press: Cambridge, UK, 1997; p. 131.

30. Perry, C.J.; Parveen, Z. The cyclisation of substituted phthalanilic acids in acetic acid solution: A kinetic study of substituted $N$-phenylphthalimide formation. J. Chem. Soc. Perkin Trans. 2 2001, $512-521$.

31. Tanaka, H.; Iwata, Y.; Takahashi, D.; Adachi, M.; Takahashi, T. Efficient stereoselective synthesis of $\gamma-N$-glycosyl asparagines by $\mathrm{N}$-glycosylation of primary amide groups. J. Am. Chem. Soc. 2005, 127, 1630-1631.

32. Bacchi, A.; Costa, M.; Della Cà, N.; Gabriele, B.; Salerno, G.; Cassoni, S. Heterocyclic derivative syntheses by palladium-catalyzed oxidative cyclization-alkoxycarbonylation of substituted -oxoalkynes. J. Org. Chem. 2005, 70, 4971-4979.

33. Pathak, S.; Kundu, A.; Pramanik, A. Regioselective synthesis of two types of highly substituted 2-pyridones through similar multicomponent reactions. Tetrahedron Lett. 2012, 53, 3030-3034.

34. Panda, N.; Mothkuri, R. Stereoselective synthesis of enamides by Pd-catalyzed hydroamidation of electron deficient terminal alkynes. J. Org. Chem. 2012, 77, 9407-9412.

35. Powell, M.F. A compendium and hydropathy/flexibility analysis of common reactive sites in proteins: Reactivity at Asn, Asp, Gln, and Met motifs in neutral pH solution. In Formulation, Characterization, and Stability of Protein Drugs; Pearlman, R., Wang, Y.J., Eds.; Springer Science + Business Media: New York, NY, USA, 1996; pp. 1-140.

36. Cacia, J.; Keck, R.; Presta, L.G.; Frenz, J. Isomerization of an aspartic acid residue in the complementarity-determining regions of a recombinant antibody to human IgE: Identification and effect on binding affinity. Biochemistry 1996, 35, 1897-1903.

37. Wang, W. Instability, stabilization, and formulation of liquid protein pharmaceuticals. Int. J. Pharm. 1999, 185, 129-188.

38. Harris, R.J.; Kabakoff, B.; Macchi, F.D.; Shen, F.J.; Kwong, M.; Andya, J.D.; Shire, S.J.; Bjork, N.; Totpal, K.; Chen, A.B. Identification of multiple sources of charge heterogeneity in a recombinant antibody. J. Chromatogr. B 2001, 752, 233-245.

39. Wakankar, A.A.; Borchardt, R.T. Formulation considerations for proteins susceptible to asparagine deamidation and aspartate isomerization. J. Pharm. Sci. 2006, 95, 2321-2336.

40. Wakankar, A.A.; Borchardt, R.T.; Eigenbrot, C.; Shia, S.; Wang, Y.J.; Shire, S.J.; Liu, J.L. Aspartate isomerization in the complementarity-determining regions of two closely related monoclonal antibodies. Biochemistry 2007, 46, 1534-1544. 
41. Xiao, G.; Bondarenko, P.V.; Jacob, J.; Chu, G.C.; Chelius, D. ${ }^{18}$ O labeling method for identification and quantification of succinimide in proteins. Anal. Chem. 2007, 79, 2714-2721.

42. Chu, G.C.; Chelius, D.; Xiao, G.; Khor, H.K.; Coulibaly, S.; Bondarenko, P.V. Accumulation of succinimide in a recombinant monoclonal antibody in mildly acidic buffers under elevated temperatures. Pharm. Res. 2007, 24, 1145-1156.

43. Xiao, G.; Bondarenko, P.V. Identification and quantification of degradations in the Asp-Asp motifs of a recombinant monoclonal antibody. J. Pharm. Biomed. Anal. 2008, 47, $23-30$.

44. Valliere-Douglass, J.; Jones, L.; Shpektor, D.; Kodama, P.; Wallace, A.; Balland, A.; Bailey, R.; Zhang, Y. Separation and characterization of an IgG2 antibody containing a cyclic imide in CDR1 of light chain by hydrophobic interaction chromatography and mass spectrometry. Anal. Chem. 2008, 80, 3168-3174.

45. Huang, H.Z.; Nichols, A.; Liu, D. Direct identification and quantification of aspartyl succinimide in an IgG2 mAb by RapiGest assisted digestion. Anal. Chem. 2009, 81, 1686-1692.

46. Zhang, J.; Yip, H.; Katta, V. Identification of isomerization and racemization of aspartate in the Asp-Asp motifs of a therapeutic protein. Anal. Biochem. 2011, 410, 234-243.

47. Yu, X.C.; Joe, K.; Zhang, Y.; Adriano, A.; Wang, Y.; Gazzano-Santoro, H.; Keck, R.G.; Deperalta, G.; Ling, V. Accurate determination of succinimide degradation products using high fidelity trypsin digestion peptide map analysis. Anal. Chem. 2011, 83, 5912-5919.

48. Sreedhara, A.; Cordoba, A.; Zhu, Q.; Kwong, J.; Liu, J. Characterization of the isomerization products of aspartate residues at two different sites in a monoclonal antibody. Pharm. Res. 2012, 29, 187-197.

49. Yi, L.; Beckley, N.; Gikanga, B.; Zhang, J.; Wang, Y.J.; Chih, H.W.; Sharma, V.K. Isomerization of Asp-Asp motif in model peptides and a monoclonal antibody Fab fragment. J. Pharm. Sci. 2013, 102, 947-959.

50. Herman, A.C.; Boone, T.C.; Lu, H.S. Characterization, formulation, and stability of Neupogen ${ }^{\circledR}$ (Filgrastim), a recombinant human granulocyte-colony stimulating factor. In Formulation, Characterization, and Stability of Protein Drugs; Pearlman, R., Wang, Y.J., Eds.; Springer Science + Business Media: New York, NY, USA, 1996; pp. 303-328.

51. Lam, X.M.; Yang, J.Y.; Cleland, J.L. Antioxidants for prevention of methionine oxidation in recombinant monoclonal antibody HER2. J. Pharm. Sci. 1997, 86, 1250-1255.

52. Shahrokh, Z.; Eberlein, G.; Buckley, D.; Paranandi, M.V.; Aswad, D.W.; Stratton, P.; Mischak, R.; Wang, Y.J. Major degradation products of basic fibroblast growth factor: Detection of succinimide and iso-aspartate in place of aspartate ${ }^{15}$. Pharm. Res. 1994, 11, 936-944.

53. Xie, M.; Vander Velde, D.; Morton, M.; Borchardt, R.T.; Schowen, R.L. pH-Induced change in the rate-determining step for the hydrolysis of the Asp/Asn-derived cyclic-imide intermediate in protein degradation. J. Am. Chem. Soc. 1996, 118, 8955-8956.

54. Violand, B.N.; Schlittler, M.R.; Kolodziej, E.W.; Toren, P.C.; Cabonce, M.A.; Siegel, N.R.; Duffin, K.L.; Zobel, J.F.; Smith, C.E.; Tou, J.S. Isolation and characterization of porcine somatotropin containing a succinimide residue in place of aspartate ${ }^{129}$. Protein Sci. 1992, 1 , 1634-1641. 
55. Weber, M.; Frey, W.; Peters, R. Asymmetric palladium(II)-catalyzed cascade reaction giving quaternary amino succinimides by 1,4-addition and a Nef-type reaction. Angew. Chem. Int. Ed. 2013, 52, 13223-13227.

56. Marenich, A.V.; Olson, R.M.; Kelly, C.P.; Cramer, C.J.; Truhlar, D.G. Self-consistent reaction field model for aqueous and nonaqueous solutions based on accurate polarized partial charges. $J$. Chem. Theory Comput. 2007, 3, 2011-2033.

57. Cramer, C.J.; Truhlar, D.G. A universal approach to solvation modeling. Acc. Chem. Res. 2008, 41, 760-768.

58. Ben-Naim, A.; Marcus, Y. Solvation thermodynamics of nonionic solutes. J. Chem. Phys. 1984, 81, 2016-2027.

59. Kim, Y. Direct dynamics calculation for the double proton transfer in formic acid dimer. J. Am. Chem. Soc. 1996, 118, 1522-1528.

60. Loerting, T.; Liedl, K.R. Toward elimination of discrepancies between theory and experiment: Double proton transfer in dimers of carboxylic acids. J. Am. Chem. Soc. 1998, 120, 12595-12600.

61. Durlak, P.; Berski, S.; Latajka, Z. Car-Parrinello and path integral molecular dynamics study of the hydrogen bond in the acetic acid dimer in the gas phase. J. Mol. Model. 2011, 17, 2995-3004.

62. Chou, P.T.; Chen, Y.C.; Wei, C.Y.; Chen, W.S. Excited-state amino-imino double-proton tautomerism in adenine nucleotide analogues catalyzed by carboxylic acids. J. Am. Chem. Soc. 2000, 122, 9322-9323.

63. Hung, F.T.; Hu, W.P.; Chou, P.T. The ground- and excited-state $\left({ }^{1} n \pi^{*}\right.$ and $\left.{ }^{1} \pi \pi^{*}\right)$ carboxylic acid-catalyzed proton (hydrogen atom)-transfer energy surfaces in 3-formyl-7-azaindole. J. Phys. Chem. A 2001, 105, 10475-10482.

64. Sikorska, E.; Khmelinskii, I.; Hoffmann, M.; Machado, I.F.; Ferreira, L.F.V.; Dobek, K.; Karolczak, J.; Krawczyk, A.; Insińska-Rak, M.; Sikorski, M. Ground- and excited-state double proton transfer in lumichrome/acetic acid system: Theoretical and experimental approach. J. Phys. Chem. A 2005, 109, 11707-11714.

65. Chai, S.; Zhao, G.J.; Song, P.; Yang, S.Q.; Liu, J.Y.; Han, K.L. Reconsideration of the excited-state double proton transfer (ESDPT) in 2-aminopyridine/acid systems: Role of the intermolecular hydrogen bonding in excited states. Phys. Chem. Chem. Phys. 2009, 11, 4385-4390.

66. Catak, S.; Monard, G.; Aviyente, V.; Ruiz-López, M.F. Reaction mechanism of deamidation of asparaginyl residues in peptides: Effect of solvent molecules. J. Phys. Chem. A 2006, 110, 8354-8365.

67. Spartan'14, version 1.1.4; Wavefunction, Inc.: Irvine, CA, USA, 2014.

68. Connolly, B.D.; Tran, B.; Moore, J.M.R.; Sharma, V.K.; Kosky, A. Specific catalysis of asparaginyl deamidation by carboxylic acids: Kinetic, thermodynamic, and quantitative structure-property relationship analyses. Mol. Pharm. 2014, 11, 1345-1358.

(C) 2015 by the authors; licensee MDPI, Basel, Switzerland. This article is an open access article distributed under the terms and conditions of the Creative Commons Attribution license (http://creativecommons.org/licenses/by/4.0/). 